\title{
The Ventral Visual Pathway Represents Animal Appearance over Animacy, Unlike Human Behavior and Deep Neural Networks
}

\author{
[DStefania Bracci, ${ }^{1}$ 이. Brendan Ritchie, ${ }^{1}$ Ioannis Kalfas, ${ }^{2}$ and ${ }^{\circledR}$ Hans P. Op de Beeck ${ }^{1}$ \\ ${ }^{1}$ Laboratory of Biological Psychology, and ${ }^{2}$ Laboratory of Neurophysiology and Psychophysiology, Department of Neurosciences, KU Leuven, Leuven 3000, \\ Belgium
}

Recent studies showed agreement between how the human brain and neural networks represent objects, suggesting that we might start to understand the underlying computations. However, we know that the human brain is prone to biases at many perceptual and cognitive levels, often shaped by learning history and evolutionary constraints. Here, we explore one such perceptual phenomenon, perceiving animacy, and use the performance of neural networks as a benchmark. We performed an fMRI study that dissociated object appearance (what an object looks like) from object category (animate or inanimate) by constructing a stimulus set that includes animate objects (e.g., a cow), typical inanimate objects (e.g., a mug), and, crucially, inanimate objects that look like the animate objects (e.g., a cow mug). Behavioral judgments and deep neural networks categorized images mainly by animacy, setting all objects (lookalike and inanimate) apart from the animate ones. In contrast, activity patterns in ventral occipitotemporal cortex (VTC) were better explained by object appearance: animals and lookalikes were similarly represented and separated from the inanimate objects. Furthermore, the appearance of an object interfered with proper object identification, such as failing to signal that a cow mug is a mug. The preference in VTC to represent a lookalike as animate was even present when participants performed a task requiring them to report the lookalikes as inanimate. In conclusion, VTC representations, in contrast to neural networks, fail to represent objects when visual appearance is dissociated from animacy, probably due to a preferred processing of visual features typical of animate objects.

Key words: animacy; deep neural networks; fMRI; MVPA; object representations; occipitotemporal cortex

Significance Statement

How does the brain represent objects that we perceive around us? Recent advances in artificial intelligence have suggested that object categorization and its neural correlates have now been approximated by neural networks. Here, we show that neural networks can predict animacy according to human behavior but do not explain visual cortex representations. In ventral occipitotemporal cortex, neural activity patterns were strongly biased toward object appearance, to the extent that objects with visual features resembling animals were represented closely to real animals and separated from other objects from the same category. This organization that privileges animals and their features over objects might be the result of learning history and evolutionary constraints.

\section{Introduction}

A fundamental goal in visual neuroscience is to reach a deep understanding of the neural code underlying object representa-

Received July 5, 2018; revised April 9, 2019; accepted May 6, 2019.

Author contributions: S.B. and H.P.O.d.B. designed research; S.B. performed research; S.B. analyzed data; S.B., I.K., and H.P.O.d.B. wrote the first draft of the paper; S.B., J.B.R., and H.P.O.d.B. edited the paper; S.B. and H.P.O.d.B. wrote the paper; J.B.R. and I.K. contributed unpublished reagents/analytic tools.

This was supported by the FWO (Fonds Wetenschappelijk Onderzoek) through a postdoctoral fellowship (12S1317N) and a Research Grant (1505518N to S.B.). J.B.R was founded by FWO and Horizon 2020 via a FWO [PEGASUS]2 Marie Skłodowska-Curie fellowship (12T9217N). H.P.0.d.B. was supported by the European Research Council (ERC-2011-StG-284101), a federal research action (IUAP-P7/11), the KU Leuven Research Council (C14/16/ 031), and a Hercules grant ZW11_10, and an Excellence of Science grant (grant number G0E8718N). tions - how does the brain represent objects we perceive around us? Over the years, research has characterized object representations in the primate brain in terms of their content for a wide range of visual and semantic object properties such as shape, size, or animacy (Konkle and Oliva, 2012; Nasr et al., 2014; Bracci and Op de Beeck, 2016; Kalfas et al., 2017). More recently, our understanding of these multidimensional object representations has been lifted to a higher level by the advent of so-called deep-

The authors declare no competing financial interests.

Correspondence should be addressed to Stefania Bracci at stefania.bracci@kuleuven.be.

https://doi.org/10.1523/JNEUROSCI.1714-18.2019

Copyright $\odot 2019$ the authors 
A

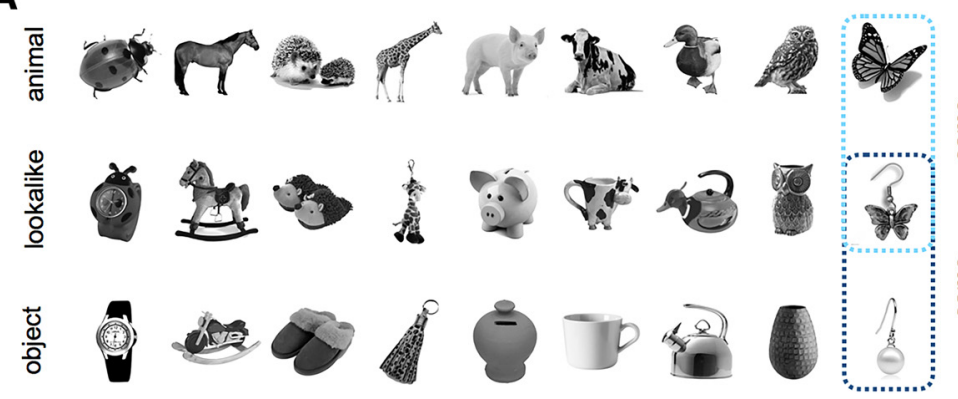

B

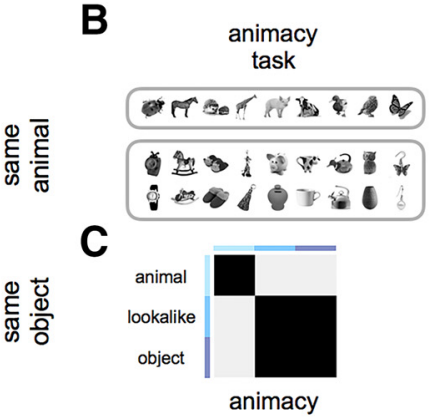

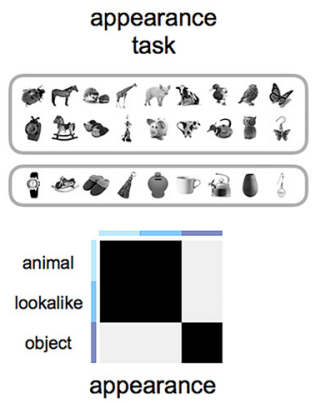

Figure 1. Experimental design. $\boldsymbol{A}$, The stimulus set was specifically designed to dissociate object appearance from object identity. We included nine different object triads. Each triad included an animal (e.g., butterfly), an inanimate object (e.g., earring), and a lookalike object closely matched to the inanimate object in terms of object identity and to the living animal in terms of object appearance (e.g., a butterfly-shaped earring). B, During fMRI acquisition, participants performed two different tasks counterbalanced across runs. During the animacy task, participants judged animacy: "does this image depict a living animal?" During the animal appearance task, participants judged animal resemblance: "does this image look like an animal?" Participants responded "yes" or " $\mathrm{no}$ " with the index and middle finger. Responses were counterbalanced across runs. C, Model predictions represent the required response similarity in the two tasks. The animacy model predicts high similarity among images that share semantic living/animate properties, thus predicting all inanimate objects (objects and lookalikes) to cluster together and separately from living animals. Conversely, the animal appearance model predicts similarities based on visual appearance despite differences in object identity and animacy, thus predicting lookalikes and animals to cluster together and separately from inanimate objects. The two models are independent $(r=0.07)$.

convolutional neural networks (DNNs) that do not only reach human behavioral performance in image categorization (Russakovsky et al., 2014; He et al., 2015; Kheradpisheh et al., 2016a), but also appear to develop representations that share many of the properties of primate object representations (Cadieu et al., 2014; Güçlü and van Gerven, 2014; Khaligh-Razavi and Kriegeskorte, 2014; Yamins et al., 2014; Güçlü and van Gerven, 2015; Kubilius et al., 2016). This correspondence extends to the representation of several object dimensions, such as shape properties (Kubilius et al., 2016) and the distinction between animate and inanimate objects (Khaligh-Razavi and Kriegeskorte, 2014). Therefore, the availability of computational models that can mimic human recognition behavior and neural information processing in its full complexity offers exciting possibilities for understanding human object vision (Kriegeskorte, 2015). Here, we provide an important test of this similarity between artificial and biological brain representations by focusing upon a particularly challenging organizing principle of human ventral visual cortex: object animacy.

Perception of animacy has played an essential role through the evolution and survival of our species. This dominant role of animacy is evident in both bottom-up perceptually driven contexts (Gao et al., 2009, 2010; Scholl and Gao, 2013), even after controlling for stimulus low-level visual properties (New et al., 2007), as well as more high-level cognitive phenomena such as pareidolia, in which animate objects (faces or animals) are most often perceived in meaningless, random noise images (e.g., clouds). At the neural level, our visual system includes specific neural mechanisms with selectivity for animate entities, such as animals and humans, relative to inanimate objects (Kanwisher et al., 1997; Downing et al., 2001). What are the dimensions underlying animacy percepts? One proposal suggests that animacy representations in visual cortex reflect the psychological dimension of perceiving something as being a living entity (Caramazza and Shelton, 1998; Tremoulet and Feldman, 2000; Gao et al., 2009). Alternatively, animacy representations might be "not aware" of object animacy per se but instead reflect stimulus visual aspects such as appearance; that is, whether something looks like a living entity or not. Here, we tested these two alternatives and investigated whether the representation of animacy converges across artificial and biological brains.

We explicitly dissociated object appearance (how an object looks) from object category (what the object really is), two di- mensions that are typically correlated in natural images, to test whether (1) both biological and artificial brains represent animacy in the same way and (2) animacy percepts reflect object appearance or object category. We show that activity patterns in visual cortex reflected animal appearance: a cow-shaped mug was more similar to a cow as opposed to a mug. In contrast, DNNs correctly categorized a cow mug as an inanimate object. As a consequence, rather surprisingly, DNNs, which were never explicitly trained on animacy, outperform ventral occipitotemporal cortex (VTC) representations in categorizing objects as being animate or inanimate.

\section{Materials and Methods \\ Participants}

The study included 17 adult volunteers ( 9 males; mean age, 30 years). Informed consent to take part in the fMRI experiment was signed by all participants. The ethics committee of the KU Leuven approved the study. For the fMRI experiment, due to excessive head motion, all data from one participant were excluded. In addition, one run was excluded in two participants and two runs were excluded in two other participants. The head motion exclusion criterion was set to $\pm 3 \mathrm{~mm}$ (equal to 1 voxel size) and defined before data collection. For behavioral ratings, two participants were excluded due to technical problems during data collection.

\section{Stimuli}

The stimulus set included nine different triads (27 stimuli in total), each containing the following: (1) one animal (e.g., a cow), (2) one object (e.g., a mug), and (3) one lookalike object that resembled the animal (e.g., a cow-shaped mug; Fig. 1A). Critically, to dissociate object appearance from object identity, each stimulus in the lookalike condition was matched to the inanimate objects in terms of object identity and to the animals in terms of animal appearance. That is, the lookalike and object conditions shared the same object identity, size, and other object properties such as function and usage (e.g., the mug and the cow mug). At the same time, the lookalike and animal conditions shared animal appearance, but differed in animacy; the cow mug is an artifact whereas the cow depicts a living animal. This stimulus set was used to acquire behavioral, DNN, and neuroimaging data.

\section{Experimental design}

Behavioral data

Each participant rated all images (Fig. 1A) based on their general similarity. Images were simultaneously rated on a screen within a circle arena following the procedure of Kriegeskorte and Mur (2012). Participants 
were asked to "arrange the objects according to how similar they are," thus letting them be free to choose what they considered to be the most relevant dimension/s to judge. The object dissimilarity space for behavioral judgments was constructed by averaging results across participants. Only the upper triangle of the dissimilarity matrix was used for subsequent analyses.

\section{DNN data}

To create the object dissimilarity space of our stimulus set, we used the stimuli features that were extracted for the last processing stage (last fully connected layer) of two DNNs, VGG-19 (Simonyan and Zisserman, 2014) and GoogLeNet (Szegedy et al., 2015), which is supposedly the best candidate layer for VTC representations. As for behavioral data, only the upper triangle of the dissimilarity matrix was used for subsequent analyses. These DNNs are deep convolutional neural networks, which have been very successful for object recognition tasks in the last few years. They consist of various processing stages, which are often termed as 'layers' either individually or in groups, in a feedforward manner that nonlinearly transform an input image volume (width, height, RGB value) into a $1 \mathrm{D}$ vector containing the class scores.

These processing stages include: (1) convolutional layers, where a neuron outputs the dot product between a kernel (its weights) and a small region in the input it is connected to; (2) rectified linear unit activation functions, such that the activations of a previous weight layer are thresholded at zero $[\max (0, x)]$; (3) max pooling layers performing a downsampling operation along the width and height of the input; and last (4) fully connected layers (resembling a multilayer perceptron) that flatten the previous stage's volume and end up in a $1 \mathrm{D}$ vector with the same size as the number of classes. A softmax function is then typically applied to this last fully connected layer's unit activations to retrieve class probability scores for the classification task. In our experiments, we used the last fully connected layer as the upper layer of the network and not the probability scores from applying the softmax function.

The two networks were trained on $\sim 1.2$ million natural images belonging to 1000 classes for the ImageNet Large Scale Visual Recognition Challenge 2012 (ILSVRC2012). The classes include $\sim 40 \%$ animals and $\sim 60 \%$ objects, but the networks are not specifically trained to detect animacy. We used the pretrained models from MatConvNet (http:// www.vlfeat.org/matconvnet/) (Vedaldi and Lenc, 2016) toolbox in MATLAB. Before feature extraction, the mean of the ILSVRC2012 training images was subtracted from each image, as per the standards of training for both of these DNNs. Furthermore, all stimuli were scaled to $224 \times 224$ pixels, in accordance with the requirements of the two networks.

$V G G$-19. This DNN from Simonyan and Zisserman (2014) incorporates 16 convolutional layers in two blocks of two convolutional layers and three blocks of four convolutional layers, followed by three fully connected layers. A max pooling operation is applied after each of these five convolutional layer blocks. All, except for the last ( $\mathrm{fc} 8$ ), of these weight layers are followed by a RELU function, where the activations are thresholded at zero. A softmax function is then applied to the last fully connected layer ( $\mathrm{fc} 8$ ). The top-5 error rate performance of this pretrained MatConvNet model on the ILSVRC2012 validation data was $9.9 \%$.

GoogLeNet. Google's entry to ILSVRC2014 (Szegedy et al., 2015) made use of the "inception" module, which is a technique used in early versions of DNNs for pattern recognition in which a DNN uses several sizes of kernels along with pooling concatenated within one layer, which is similar to integrating all information about parts of the image (size, location, texture, etc.). In addition, there is a softmax operation in multiple stages of GoogLeNet, assisting the classification procedure during training along the depth levels of the network. This MatConvNet pretrained model was imported from the Princeton version, not by the Google team, thus there is some difference in performance to other versions probably due to parameter settings during training. The top- 5 error rate performance of this model on the ILSVRC2012 validation data was 12.9\%.

\section{fMRI data}

Experimental design. We acquired neuroimaging data by means of an event-related design in two separated sessions, each performed in sepa- rated days with no more than $7 \mathrm{~d}$ between the first and second session. Each session included six experimental runs as well as additional runs with unrelated stimuli for another experiment (not reported here). The stimuli presentation was controlled by a PC running the Psychophysics Toolbox package (Brainard, 1997) in MATLAB (The MathWorks). Pictures from the stimulus set were projected onto a screen and were viewed through a mirror mounted on the head coil.

Each experimental run (12 in total) lasted $7 \mathrm{~min}$ and $14 \mathrm{~ms}(230$ volumes per run). For each subject and for each run, a fully randomized sequence of 27 image trials (repeated 4 times) and 9 fixation trials (repeated 4 times) was presented. Each trial was presented for $1500 \mathrm{~ms}$, followed by a fixation screen for $1500 \mathrm{~ms}$. Each run started and ended with $14 \mathrm{~s}$ of fixation. During the whole experiment, each stimulus was repeated 48 times. While scanning, participants performed two different tasks (Fig. $1 B$ ) counterbalanced across runs. During the animacy task, participants judged animacy (“does this image depict a living animal?”). During the appearance task, participants judged object appearance ("does this image look like an animal?"). Participants responded "yes" or "no" with the index and middle finger. Response-finger associations were counterbalanced across runs.

Acquisition parameters. Imaging data was acquired on a 3T Philips scanner with a 32-channel coil at the Department of Radiology of the University Hospitals Leuven. MRI volumes were collected using echoplanar (EPI) T2*-weighted scans. Acquisition parameters were as follows: repetition time (TR) of $2 \mathrm{~s}$, echo time (TE) of $30 \mathrm{~ms}$, flip angle (FA) of $90^{\circ}$, field of view (FoV) of $216 \mathrm{~mm}$, and matrix size of $72 \times 72$. Each volume comprised 37 axial slices (covering the whole brain) with $3 \mathrm{~mm}$ thickness and no gap. The T1-weighted anatomical images were acquired with an MP-RAGE sequence, with $1 \times 1 \times 1 \mathrm{~mm}$ resolution.

Preprocessing. Imaging data were preprocessed and analyzed with the Statistical Parametrical Mapping software package (SPM 12, Welcome Department of Cognitive Neurology) and MATLAB. Before statistical analysis, functional images underwent a standard preprocessing procedure to align, coregister and normalize to an MNI (Montreal Neurological Institute) template. Functional images were spatially smoothed by convolution of a Gaussian kernel of $4 \mathrm{~mm}$ full width at half-maximum (Op de Beeck, 2010). For each participant, a general linear model (GLM) for both tasks was created to model the 27 conditions of interest and the six motion correction parameters ( $x, y, z$ for translation and for rotation). Each predictor's time course was modeled for $3 \mathrm{~s}$ (stimulus presentation + fixation) by a boxcar function convolved with the canonical hemodynamic response function in SPM. We also analyzed data for each task separately, for which purpose a GLM was created for each task separately.

Regions of interest (ROIs) definition. ROIs were defined at the group level with a combination of functional and anatomical criteria. First, we selected all visually active voxels (all stimuli versus baseline) that exceeded the statistical uncorrected threshold $p<0.001$. Subsequently, we selected all spatially continuous voxels within anatomical ROIs defined with the Neuromorphometrics atlas in SPM. The following ROIs were defined: V1 and VTC divided into its posterior (post-VTC) and anterior (ant-VTC) sectors. These ROIs were chosen based on the important role played by VTC in object representation and categorization (Grill-Spector and Weiner, 2014). Importantly, no difference in mean response for animals and objects was observed in our ROIs (post-VTC: $p=0.12$; ant-VTC: $p=0.65$ ), showing that the ROIs were not biased toward either animate or inanimate representations. Instead, significantly higher response was observed for lookalikes relative to both animals and objects ( $p<0.001$ for both conditions and ROIs). This higher response for lookalikes is likely explained by taking into account task difficulty: response latency for lookalikes was significantly longer in both tasks $(p<$ 0.0001 , for both conditions).

\section{Statistical analyses}

Multivariate analyses were used to investigate the extent to which the two experimental dimensions (object animacy and object appearance) explain representational content in behavioral, DNNs, and brain data (this latter under different task conditions). For statistical tests, we took the following approach. For DNN models, given that only one similarity matrix for each model was available, statistical significance in the differ- 
ent analyses (e.g., representational similarity analysis) was tested across stimuli with permutation tests. To be consistent, this approach was also applied to the behavioral data (i.e., these two datasets were directly compared). For neural data, instead, given that individual subject data was available, we tested significance across subjects with ANOVAs and pairwise $t$ tests. Whereas corrections for multiple comparisons were applied for all analyses; for transparency, throughout the text, uncorrected $p$-values are reported. However, when $p$-values did not survive correction, we have noted it in the text.

ROI-based RSA. As described previously (Op de Beeck et al., 2010), for each voxel within a given ROI, parameter estimates for each condition (relative to baseline) were extracted for each participant and each run and normalized by subtracting the mean response across all conditions. Subsequently, the dataset was divided 100 times into two random subsets of runs (set- 1 and set-2) and the voxel response patterns for each object pair were correlated across these independent datasets. Correlations were averaged across the 100 iterations, resulting in an asymmetric $27 \times 27$ correlation matrix for each task, participant and ROI. For each correlation matrix, cells above and below the diagonal were averaged and only the upper triangle of the resulting symmetric matrix was used in the subsequent analyses (Ritchie et al., 2017). Correlation matrices were converted into dissimilarities matrices ( 1 - Pearson's $r$ ) and used as neural input for subsequent analyses. Resulting correlations were Fisher transformed $\left\{0.5^{*} \log [(1+\mathrm{r}) /(1-\mathrm{r})]\right\}$ and tested with ANOVAs and pairwise $t$ tests.

For each ROI, we computed an estimate of the reliability of the dissimilarity matrices (Op de Beeck et al., 2008), which indicates the highest expected correlation in a region given its signal-to-noise ratio. For each subject and each ROI, the $27 \times 27$ correlation matrix was correlated with the averaged correlation matrix of the remaining participants. The resulting correlation values (averaged across participants) capture noise inherent to a single subject as well as noise caused by intersubject variability.

Whole-brain RSA. In addition to ROI-based RSA, we performed a whole-brain RSA correlating the two models (appearance, animacy) with neural patterns throughout the brain. The whole-brain RSA performed using the volume-based searching approach (Kriegeskorte et al., 2006) was implemented with CoSMo MVPA (Oosterhof et al., 2016). Parameter estimates for each condition (relative to baseline) were extracted for each participant and each run and normalized by subtracting the mean response across all conditions. Resulting values were then averaged across all runs. For each voxel in the brain, a searchlight was defined using a spherical neighborhood with a variable radius, including the 100 voxels nearest to the center voxel. For each searchlight, the neural dissimilarity matrix was computed for the 27 stimuli. The neural dissimilarity matrix (upper triangle) was then correlated with the dissimilarity matrices derived from the two predictive models (Fig. 1C). The output correlation values were Fisher transformed and assigned to the center voxel of the sphere. Resulting whole-brain correlation maps for each of the models were directly contrasted and differences were tested using randomeffects whole-brain group analysis and corrected with the threshold-free cluster enhancement (TFCE) method (Smith and Nichols, 2009). Voxelwise-corrected statistical maps for each model relative to baseline $(z=1.64 ; p<0.05$, one-sided $t$ test $)$ and the direct contrast between the two predictive models ( $z=1.94 ; p<0.05$, two-sided $t$ test) are displayed on a brain template by means of BrainNet Viewer (Xia et al., 2013).

Classification analysis. As a complementary analysis to correlationbased MVPA, category discriminability and stimulus identity discriminability were tested with linear discriminant analysis (LDA) by means of the CoSMoMVPA toolbox (Oosterhof et al., 2016). For each condition and ROI, classifiers were trained and tested on the $\beta$ estimates using the leave-one-run-out cross-validation method. Category discriminability was tested with a three-way classification and values in the confusion matrix were analyzed to test confusability between categories. That is, even though a condition might be classified above chance, higher error rates with another condition is indicative of higher similarity of their representational spaces. Generalization across triads was tested with leave-one-triad-out. Stimulus identity discriminability was tested within-condition (e.g., discriminating a butterfly in the animal condi- tion) and between-condition (generalization analysis), which tested the ability of a classifier to match each item of a triad across conditions (for lookalikes only). This latter analysis tests identity generalization of each lookalike to its corresponding animal or its corresponding object.

\section{Data and software availability}

All types of brain images and statistics data are available from the authors upon request. The data used for final statistics are made available through the Open Science Framework at https://osf.io/k6qne/.

\section{Results}

We constructed a stimulus set to intentionally separate object appearance from object identity, including: nine animals (e.g., a cow), nine objects (e.g., a mug), and nine lookalike objects, which consisted of objects (e.g., a cow mug) that were matched to the inanimate objects in terms of object identity and to the animals in terms of appearance. This resulted in a stimulus set of nine closely matched triads, 27 stimuli in total (Fig. 1A). The mug and the cow mug represent the same inanimate object, identical in many respects (e.g., function, size, material, and manipulability) but their appearance. Conversely, relative to the cow mug, the cow is a living animal and despite shared visual features typical of animals, such as eyes, mouth, and limbs, differs in all the above semantic and functional properties. With this stimulus design, we dissociate the conceptual animate-inanimate distinction from visual appearance (Fig. $1 C$ ). The two models are orthogonal $(r=0.07)$.

\section{DNNs and human perception privilege object animacy over appearance}

To test whether DNNs predict human perception on object animacy and appearance, we compared similarity judgments on the stimulus set (Materials and Methods) to the stimulus representation for two recent DNNs (VGG-19: Simonyan and Zisserman, 2014; and GoogLeNet: Szegedy et al., 2015) that were chosen based on their human-like performance in object categorization (Russakovsky et al., 2014; He et al., 2015; Kheradpisheh et al., 2016b). The object dissimilarity for each image pair (1 - Pearson's $r$ ) was computed for human similarity ratings (Materials and Methods) and for the output vectors of the DNNs' last fully connected layer, and resulting dissimilarity matrices were correlated with the two independent models. Results revealed similarities but also differences between human judgments and DNNs representations (Fig. 2A). A significant positive correlation of both models (appearance: $r=0.41 ; p<0.0001$; animacy: $r=$ 0.66 ; $p<0.0001$ ) with behavioral judgments showed that participants perceived both dimensions, with a significant preference for the animacy percept $(p=0.0006)$. This preference was particularly strong in DNNs' representations, with a highly significant correlation with the animacy model (VGG-19: $r=0.73 ; p<$ 0.0001; GoogLeNet: $r=0.55$; $p<0.0001)$, but no correlation with the appearance model (VGG-19: $r=-0.04$; GoogLeNet: $r=-0.01 ; p>0.5$, for both DNNs). The preference for object animacy over appearance was present throughout all DNNs' layers (Fig. 2B). It is, however, interesting that although the appearance model is mostly below baseline throughout most layers in both networks, toward the end the networks' architecture, it reaches its peak (VGG-19: $r=0.20$; GoogLeNet: $r=0.19$ ) to subsequently drop back to baseline at the final processing stages. As a result, the magnitude of the preference for object animacy peaks in the last layer.

When inspecting dissimilarity matrices and 2D arrangements derived from multidimensional scaling (MDS) (Fig. $3 A, B$ ), animals appear to be separated from objects and lookalikes in both 
A

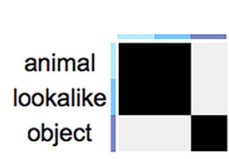

appearance

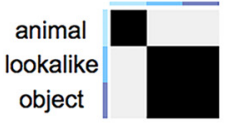

animacy behavior

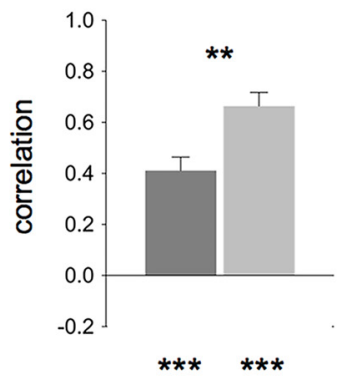

similarity judgements
B

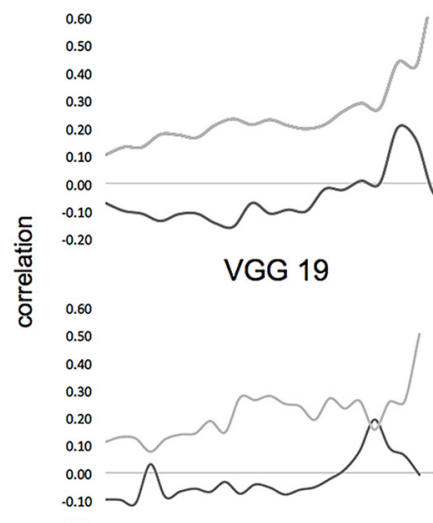

GoogLeNet

Figure 2. DNNs predict human perception of object animacy but not appearance. $A$, RSA (Kriegeskorte et al., 2008a) results for the appearance model (dark gray) and the animacy model (light gray) are shown for human judgments (left), and DNNs (right). Asterisks indicate significant values computed with permutation tests (10,000 randomizations of stimulus labels) and error bars indicate SE computed by bootstrap resampling of the stimuli. ${ }^{* * *} p<0.0001,{ }^{* *} p<0.001$. B, RSA results for the two models are shown for DNNs' individual layers.

A
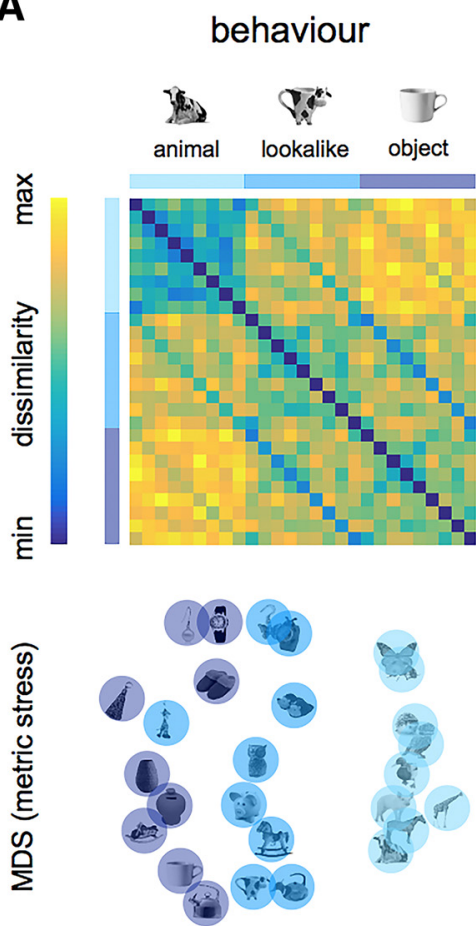

B
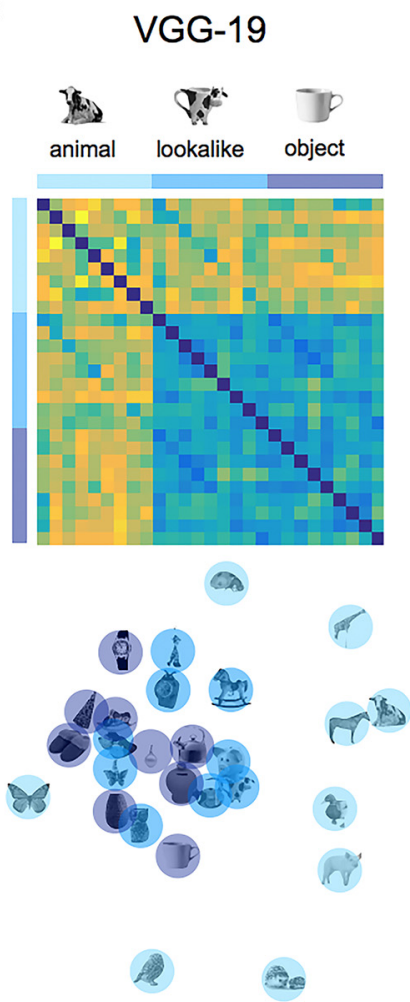

GoogLeNet
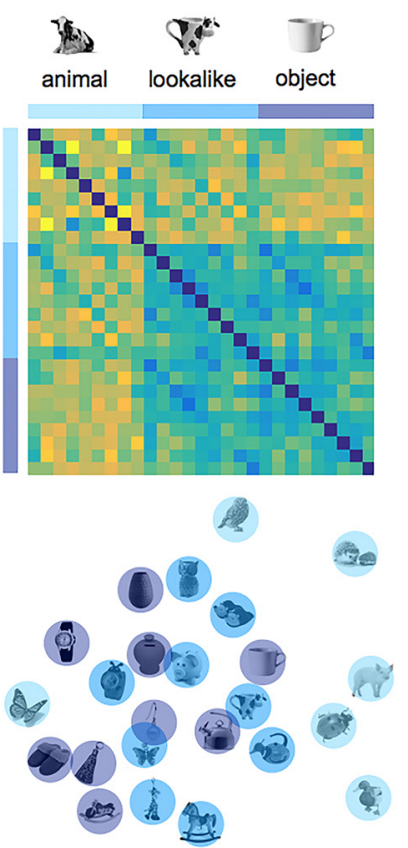

Figure 3. DNNs and human perception predict a representation based on object animacy, over appearance. $\boldsymbol{A}, \boldsymbol{B}$, Dissimilarities matrices (top) and $2 \mathrm{D}$ arrangements derived from MDS (metric stress; bottom) showing stimuli pairwise distances for behavioral judgments ( $\boldsymbol{A})$ and DNNs ( $\boldsymbol{B})$. Light blue, Animals; blue, lookalikes; dark blue, objects.

behavioral and DNN dissimilarity matrices (see also category index in Fig. 7). In addition, animals appear to cluster together in behavioral data with some additional similarities to the lookalike objects, which was absent in DNN visual arrangements. In further analyses on the dissimilarity matrices, we will test the significance of these differences between the three major conditions (animals, objects, lookalikes) in the different datasets.

Together, these data show that both humans and DNNs set apart animate from inanimate objects in accordance with one of the most reported divisions in ventral occipitotemporal cortex (VTC) (Kriegeskorte et al., 2008b; Grill-Spector and Weiner, 2014).

\section{Animal appearance explains pattern information in the} human visual cortex

The above results, from DNNs and human behavior, point to the animacy model as the representational structure to sustain object representations even with a stimulus set that sets object animacy apart from object visual appearance. Based on previous studies, 
A

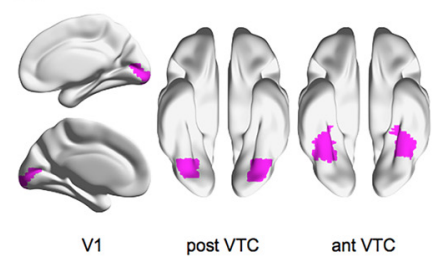

B

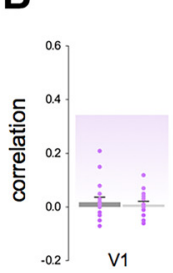

both tasks

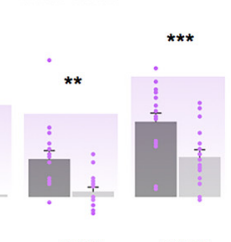

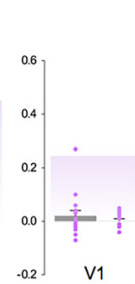
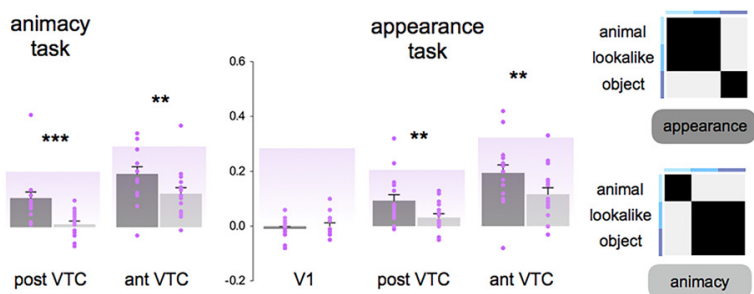

Figure 4. Animal appearance better explains representational content in human visual cortex. $A$, Group-averaged ROls (V1, post-VTC, ant-VTC) are shown on an inflated human brain template in BrainNet Viewer (Xia et al., 2013). B, RSA (Kriegeskorte et al., 2008a) results for the appearance model (dark gray) and the animacy model (light gray) are shown for the data combined across the two tasks and for each task separately. Individual participant's $(n=16)$ correlation values are shown in purple. Purple-shaded backgrounds represent reliability values of the correlational patterns taking into account the noise in the data (Materials and Methods). These values give an estimate of the highest correlation that we can expect in each ROI. Error bars indicate SEM. Asterisks indicate significant difference between the two models $\left({ }^{* * *} p<0.001 ;{ }^{* *} p<0.01\right)$.

we would expect this organizational principle to have its neural correlate in VTC (Kriegeskorte et al., 2008b; Grill-Spector and Weiner, 2014), possibly accompanied by a further effect of visual appearance (Bracci and Op de Beeck, 2016). We collected human functional neuroimaging (fMRI) data in an event-related design (see Materials and Methods). All 27 images were presented in a random order while participants performed two orthogonal tasks (matching the models) counterbalanced across runs (Fig. 1B). During the animacy task, participants judged whether the image on the screen depicted a living animal (yes or no). During the appearance task, participants judged whether the image on the screen looked like an animal (yes or no). In this way, we forced participants to group the lookalike condition in two different ways depending on their properties (Fig. 1C): either similarly to the object condition (as in the animacy model) or similarly to the animal condition (as in the appearance model). Thus, in addition to testing the two independent predictive models, we could assess any task-related modulation.

We correlated the dissimilarity values predicted by the two models with the dissimilarity matrices derived from neural activity patterns elicited in target rROIs (Fig. $4 A$ ) in visual cortex. Our main ROI was VTC, divided into the ant-VTC and the post-VTC. In addition, we included V1 as a control ROI. Analyses were performed separately for each task, but when no task-related effects were observed, we mostly focus on results for the combined dataset.

To investigate the role of animacy and animal appearance in driving the VTC organization, correlation values were tested in a $3 \times 2$ ANOVA with ROI (V1, post-VTC, ant-VTC) and model (appearance, animacy) as within-subject factors. Results revealed a significant ROI $\times$ model interaction $\left(F_{(2,30)}=9.40, p=0.001\right.$; Fig. $4 B$ ), thus highlighting differences in the relation between the two models and representational content in the three ROIs. No positive correlations were found in V1 (lookalike: $t<1$; animacy: $t<1$ ), suggesting that our stimulus set was constructed appropriately to investigate neural representations without trivial confounds with low-level visual features. Unexpectedly, and differently from DNNs and behavioral results, neural representations in ant-VTC and post-VTC were significantly more correlated with the appearance model than the animacy model (post-VTC: $t_{(15)}=3.85, p=0.002$; ant-VTC: $t_{(15)}=4.00, p=$ $0.001)$. In addition, we also observed differences between the ant-VTC and post-VTC. Whereas in ant-VTC, both models were significantly correlated with the neural data (appearance: $t_{(15)}=$ 8.70, $p<0.00001$; animacy: $t_{(15)}=5.36, p<0.00001$ ), in postVTC, only correlations with the appearance model significantly differed from baseline (lookalike: $t_{(15)}=4.56, p=0.0004$; animacy: $t<1.5)$. Replicating results within subjects, data analyzed for the two tasks separately did not reveal any task effect in VTC (Fig. 4B). In both tasks, the appearance model was significantly more correlated with the neural data as opposed to the animacy model in post-VTC (animacy task: $t_{(15)}=3.83, p=0.002$; appearance task: $t_{(15)}=3.05, p=0.008$ ) and ant-VTC (animacy task: $t_{(15)}=2.97, p=0.009$; appearance task: $t_{(15)}=3.44, p=$ 0.004). Furthermore, none of the ROIs showed an effect of task in a direct statistical comparison (ant-VTC: $F<1$; post-VTC: $F<$ 1). To visualize the representational structure in each ROI in more detail, dissimilarities matrices (averaged across tasks) MDS arrangements are shown in Figure 5, $A$ and $B$.

To test whether DNNs representations or behavioral judgments might provide better models for VTC space, we compared all five models against each other with partial correlation (appearance, animacy, similarity judgments, VGG19, and GoogLeNet). Results confirmed the above findings showing that the appearance model better predicts representational content in anterior VTC relative to all remaining models (task 1: $p<0.003$; task 2: $p<0.001$; both tasks: $p<0.00004$, for all models).

Findings from the ROI-based analysis were backed up by a whole-brain searchlight analysis. Whereas both models revealed significant effects in VTC (Fig. 6A), the direct comparison revealed a significant preference for the appearance model over the animacy model in (right) VTC $(p<0.05$, two-tailed $t$ test, TFCE corrected; Fig. 6B). The opposite contrast (animacy $>$ appearance) did not reveal any effect across the whole brain.

Together, these results show that the animacy organization reported in VTC is largely explained by animal appearance rather than object animacy per se. Inanimate objects (e.g., cow mug) that share with animals neither functional properties nor animacy, are represented closer to living animals than to other inanimate objects with which they share object category (e.g., a mug), and functional/semantic properties. The animal appearance might relate to high-level visual features such as faces, eyes, limbs, and bodies, which are not generally present in inanimate objects. This result, replicated across tasks, is particularly striking in light of the aforementioned results from human judgments and DNNs, which privilege object animacy over appearance.

\section{Do VTC representations distinguish between lookalikes and real animals?}

In anterior VTC the two (independent) models both differ from baseline. Therefore, although the lookalikes were closer to animals than to objects, lookalikes and animals might be encoded separately in VTC. Another possibility is that the positive correlation with the animacy model is fully driven by the distinction between animals and (non-lookalike) objects (which both predictive models share), without any representation of the 

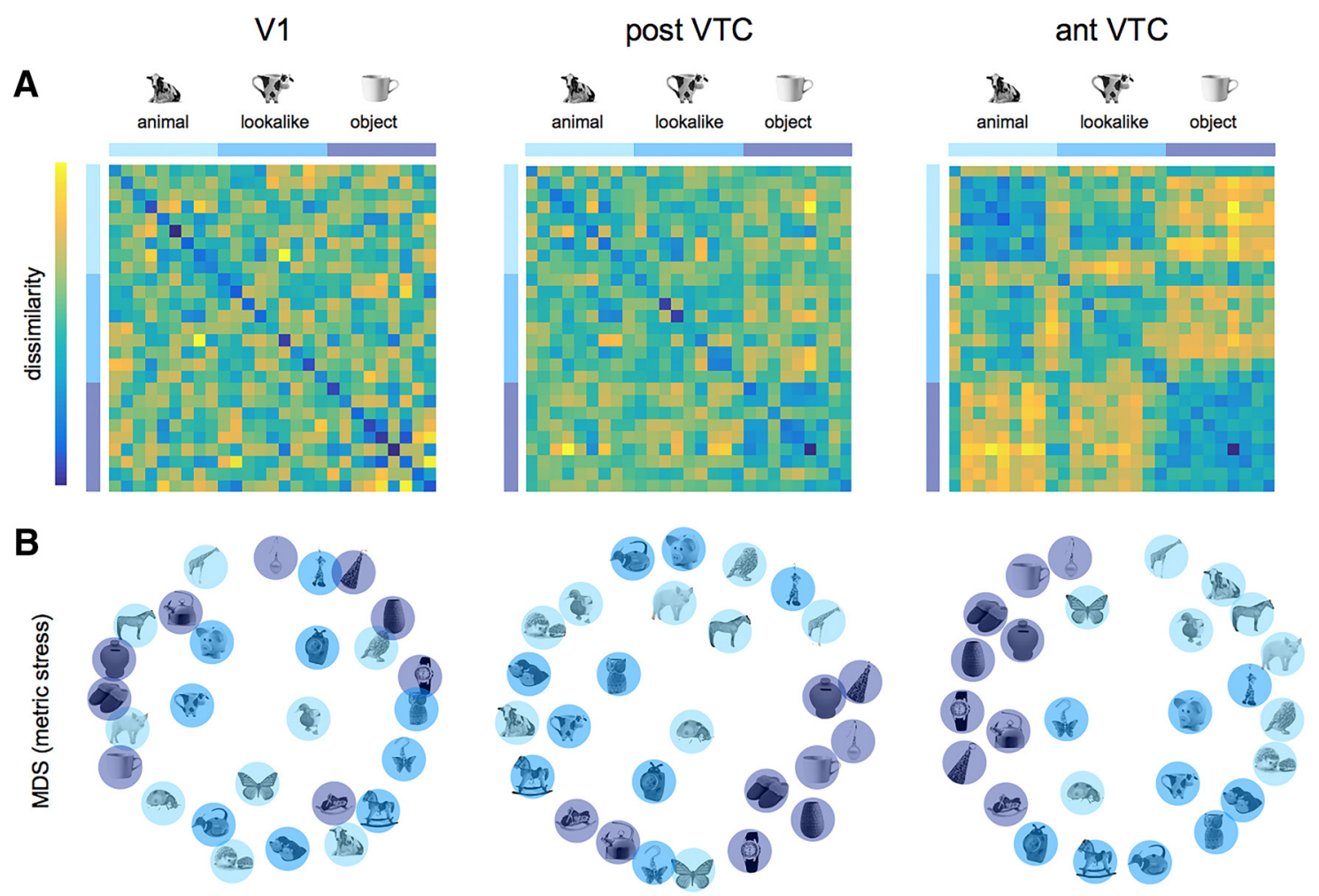

Figure 5. Neural similarity space in VTC reflects animal appearance. $A$, Neural dissimilarity matrices (1 - Person's $r$ ) derived from the neural data (averaged across subjects and tasks) showing pairwise dissimilarities among stimuli in the three ROls. B, The MDS (metric stress), performed on the dissimilarity matrices averaged across subjects and tasks, shows pairwise distances in a 2 D space for the three ROls. Light blue, Animals; blue, lookalikes; dark blue, objects.

\section{A}

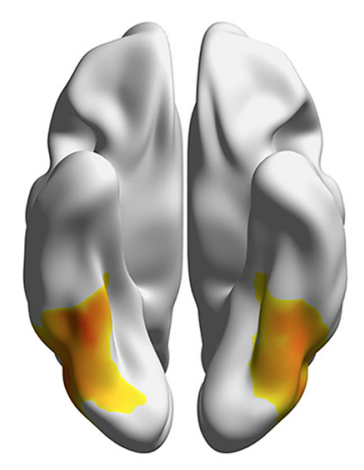

appearance

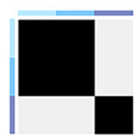

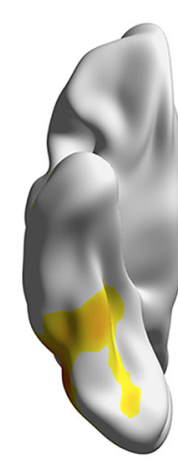

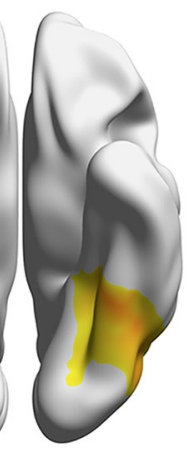

animacy
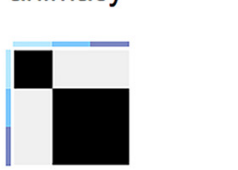

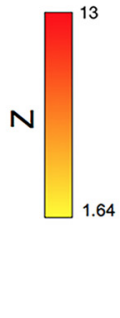

uncorrected
B
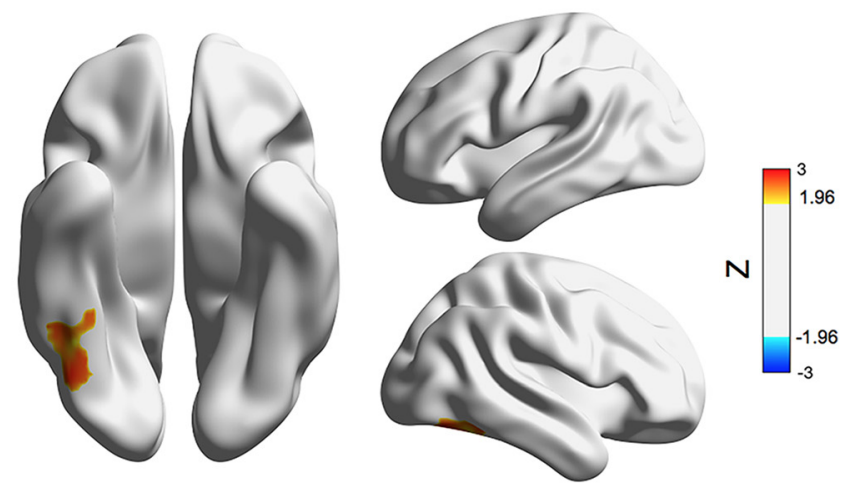

appearance vs animacy

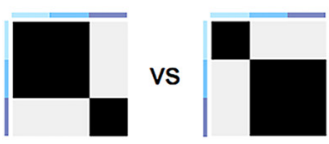

Threshold-Free Cluster Enhancement

Figure 6. Whole-brain RSA. Shown are the results of random-effects whole-brain RSA for $(\boldsymbol{A})$ individual models (uncorrected) and $(\boldsymbol{B})$ the direct contrast between the two predictive models (appearance vs animacy) corrected with the TFCE (Smith and Nichols, 2009) method are displayed on a brain template by means of BrainNet Viewer (Xia et al., 2013).

lookalikes as being different from real animals (on which the two predictive models make opposite predictions). In other words, does VTC discriminate between animals and objects that look like animals or is it largely blind to this category distinction? If VTC does not distinguish between animals and objects that have animal appearance then there should be no difference between within- and between-condition correlations for these two categories. In what follows we investigated this hypothesis.

To this aim, we computed the category index, which reflects the extent to which representations for two conditions can be discriminated from each other. That is, for each subject and condition, the average within-condition correlation (e.g., comparing 


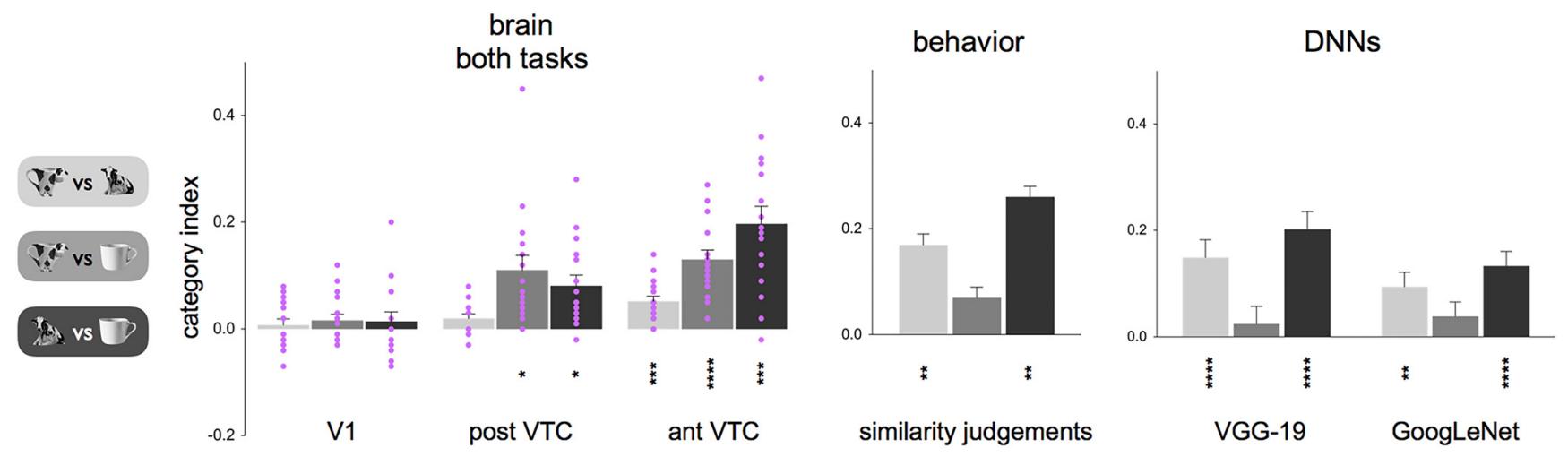

Figure 7. VTC representations differ in category discriminability from DNNs and human behavior. The category index reflects representational discriminability among the three stimulus categories (animals, lookalikes, and objects) and is computed for each condition pair by subtracting the average of between-condition correlations (e.g., for animals and lookalikes), from the average of within-condition correlations (e.g., for animals and lookalikes). Results are reported for neural data (left), behavior (middle), and DNNs (right). Light gray, Animals versus lookalikes; gray, lookalikes versus objects; dark gray, animals versus objects. For neural data, individual participant's $(n=16)$ data points are shown in purple. Asterisks indicate significant values relative to baseline and error bars indicate SEM. For behavioral and DNNs data, asterisks indicate significant values relative to baseline computed with permutation tests $(10,000$ randomizations of stimulus labels) and error bars indicate SE computed by bootstrap resampling of the stimuli. ${ }^{* * *} p<0.00001,{ }^{* * *}<p<0.0001,{ }^{* *}<p<0.001,{ }^{*}<p<0.01$.

an animal with other animals) and between-condition correlation (e.g., comparing animals with lookalikes or objects) were calculated. For each condition pair (i.e., animal-lookalike, animal-object, and lookalike-object), the category index was computed as follows: first averaging the two within-condition correlations (for animals and lookalikes) and then subtracting the between-condition correlation for the same two categories (animals-lookalikes). For between-condition correlations, diagonal values (e.g., duck and duck kettle) were excluded from this computation to avoid intrinsic bias between conditions that share either animal appearance (i.e., duck and duck kettle) or object category (i.e., kettle and duck kettle). Category indexes above baseline indicate that two category representations are separated. In both VTC regions, the category index could distinguish between animals and objects (ant-VTC: $t_{(15)}=6.00, p=0.00002$; post-VTC: $t_{(15)}=4.03, p=0.001$; Fig. 7 , left), and objects and lookalikes (ant-VTC: $t_{(15)}=7.40, p<0.00001$; post-VTC: $t_{(15)}=$ $4.02, p=0.001)$. The category index between animals and lookalikes was significant in ant-VTC $\left(t_{(15)}=5.38, p=0.00007\right)$ but did not differ from baseline (after correcting for multiple comparisons: $\mathrm{p} 0.05 / 9=p<0.005)$ in post-VTC $\left(t_{(15)}=2.34\right.$, $p=0.03)$. Furthermore, in both ROIs, the category index for animals and lookalikes was significantly smaller than the other two category indexes (ant-VTC: $t_{(15)}>3.49, p<0.004$, for both indexes; post-VTC: $t_{(15)}>3.45, p<0.005$, for both indexes). In V1 none of the conditions could be distinguished $(t<1$, for all condition pairs). As for previous analyses, these results were replicated when data were analyzed for each task separately (the category index for animals vs lookalikes was significantly smaller than the other two indexes: animacy task: $p<0.004$; appearance task: $p<0.01)$. Thus, VTC representations reflect animal appearance much more than animacy, with a small remaining difference between animals and lookalikes in the anterior part of VTC (but not posterior VTC).

Different results were observed for behavioral judgments and DNNs. In both cases, category indexes distinguished between animals and objects (behavior: $p=0.0001$; VGG-19: $p<0.00001$; GoogLeNet: $p<0.00001$ ), between animals and lookalikes (behavior: $p=0.0006$; VGG-19: $p<0.00001$; GoogLeNet: $p=$ 0.0005 ), but did not differ between lookalikes and objects (behavior: $p=0.03$ - multiple comparisons correction: $\mathrm{p} 0.05 / 3=p<$ 0.01 ; VGG-19: $p=0.46$; GoogLeNet: $p=0.15$ ). This suggests that differently from VTC representations, human judgments, and convolutional neural network take object category into account; two mugs belong to the same object category regardless of their shape.

Together, these results show that representations in VTC are not predicted either by convolutional neural networks or human judgments. Whereas the former privileges the role of animal appearance, the latter favors the role of superordinate object category such as objects versus animals.

\section{Coding for animal appearance in VTC interferes with invariant object identification.}

Previous studies have shown that VTC contains information about object identity, with a high degree of invariance for a variety of image transformations (Gross et al., 1972; Desimone et al., 1984; Grill-Spector et al., 1998, 1999). Despite the reported finding that a lookalike object is represented very differently from other objects, VTC representations might still allow for object identity identification (e.g., recognizing that a cow mug is a mug). To allow this, the representation of a lookalike object should be more similar to another object from the same basic category (e.g., a cow mug and a regular mug) than to other objects. We operationalize this as the prediction that the within-triad correlation between each lookalike and its corresponding non-lookalike object would be significantly higher than the average of correlation of this same lookalike to objects from other triads. We call this the identity index. That is, for each subject and for each lookalike object, we took the on-diagonal correlation (e.g., between the cow mug and the mug) and subtracted the average of off-diagonal correlations (e.g., between the cow mug and the remaining objects). We computed the identity index separately for animals (animal identity index: is a cow mug closer to a cow relative to other animals?) and objects (object identity index: is a cow mug closer to a mug relative to other objects?). Figure 8 shows results for the dataset combined across the two tasks.

For brain data, a test across all conditions (Fig. 8, left) revealed differences in the amount of stimulus identity information carried in the three ROIs. In V1, there was significant identity information for objects $\left(t_{(8)}=4.76, p=0.001\right)$ but not for animals $(t<1)$. This result can be explained considering differences in image low-level visual properties across conditions; objects and 
DNNs

$$
\text { pixelwise }
$$
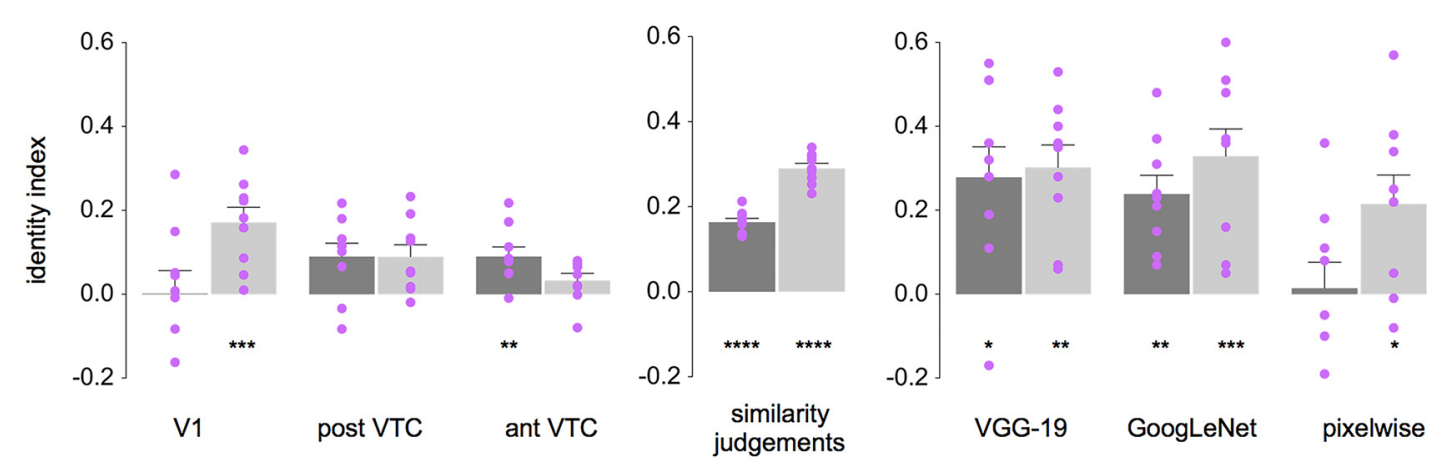

B
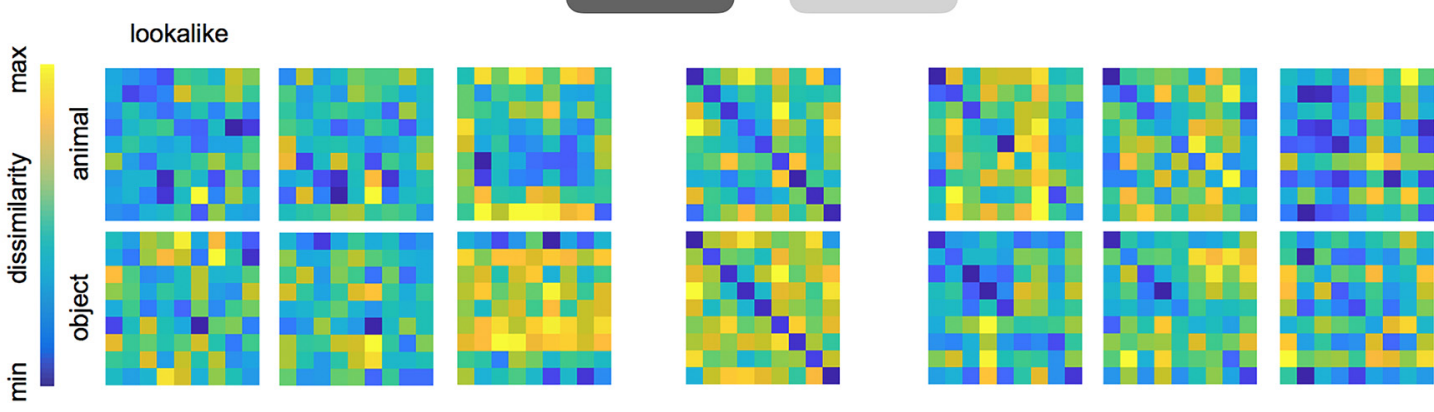

Figure 8. VTC representations sustain animal identity, but not object identity, categorization. $\boldsymbol{A}$, The identity index reflects information for individual object ad animal pairs (e.g., the cow mug and the mug represent the same object; the cow mug and the cow represent the same animal) and is computed separately for each condition (animals and objects). For each lookalike object ( $n=$ 9), we took the on-diagonal correlation (e.g., between the cow mug and the mug) and subtracted the average off-diagonal correlations (e.g., between the cow mug and the remaining objects). The identity index for animals and objects was computed for the brain data (V1, post-VTC, and ant-VTC), behavioral data (similarity judgments), DNNs (VGG-19, GoogLeNet), and the image pixelwise data. Light gray, Animal identity index; dark gray, object identity index. Asterisks $\left({ }^{* * * *} p<0.00001,{ }^{* * *}<p<0.0001,{ }^{* *}<p<0.001,{ }^{*} p<0.01\right)$ indicate significant values relative to baseline and error bars indicate SEM. $\boldsymbol{B}$, For each dataset, dissimilarity matrices used to compute the identity index are shown separately for animals and objects.

lookalikes were more similar to each other relative to animals and lookalikes. Confirming this interpretation, the same trend was observed for image pixelwise information (Fig. $8 A$, right), where we observed significant identity information for objects $\left(t_{(8)}=\right.$ $3.16, p=0.01)$ but not for animals $(t<1)$. In post-VTC, neither identity index survived correction for multiple comparisons $(\mathrm{p}$ $0.05 / 6=p<0.008$; animals: $t_{(8)}=2.82, p=0.02$; objects $t_{(8)}=$ $3.09, p=0.01)$. In ant-VTC, object identity information decreased and animal identity information increased; here, only the animal identity index was significantly above baseline (animals: $t_{(8)}=3.88, p=0.005$; objects: $t_{(8)}=1.93, p=0.09$ ). Results were replicated when data were analyzed separately for the two tasks (ant-VTC: animals, $p<0.009$; objects, $p>0.4$, for both tasks). Together, these results suggest that representational content in anterior VTC is differently biased to represent animal and object's identity, containing more information for the former.

Consistent with previous analyses, different results were observed for behavioral judgments and DNNs. For human similarity judgments, a test across conditions revealed significant identity information for both animals $\left(t_{(8)}=17.98, p<0.00001\right)$ and objects $\left(t_{(8)}=24.44, p<0.00001\right.$; Fig. $8 A$, middle). Similarly, DNNs were able to discriminate individual stimulus identities for animals $\left(\mathrm{VGG}-19: t_{(8)}=3.84, p=0.005\right.$; GoogLeNet: $t_{(8)}=5.42$, $p=0.0006)$ as well as objects (VGG-19: $t_{(8)}=5.67, p=0.0005$; GoogLeNet: $t_{(8)}=5.04, p=0.001$; Figure $8 A$, right).

Strikingly, after years of searching for models that would match the tolerance in the most advanced category representations in primate ventral visual cortex, we have now found an object transformation for which neural networks sustain invariant object recognition to a higher degree than ant-VTC representations.

\section{Results replication with decoding approach}

Recent reports suggested significant differential reliability of different dissimilarity measures (Walther et al., 2016). To test robustness of our approach, we replicated results with a different analysis: LDA (see Materials and Methods). This test confirmed full replication of our results with a different measure. An overview is reported in Figure 9.

To replicate results reported in Figure 7, category discriminability for neural data was tested with a leave-one-run-out three-way classification approach (Fig. 9A). Results in ant-VTC and post-VTC, despite revealing significant discriminability for each category ( $p<0.001$; multiple comparisons correction: $\mathrm{p}$ $0.05 / 9=p<0.005$ ), confirmed significantly less discriminability for the lookalikes relative to animals and objects (post-VTC: $t_{(15)}$ $<2.34, p<0.034$; ant-VTC: $t_{(15)}<3.82, p<0.002$, for both conditions). In ant-VTC, further analyses on the confusion matrix, confirmed higher similarities in the representational patterns for animals and lookalikes (Fig. 9A, bottom), showing higher confusability between these two conditions than between objects and lookalikes $\left(t_{(15)}=2.72, p=0.016\right)$. Next, we performed a cross-decoding analysis (Fig. $9 B$; leave-one-triad-out), in which performance depends not only upon the similarity between the three conditions within a triad, but also upon the degree of correspondence between triads. This analysis showed 

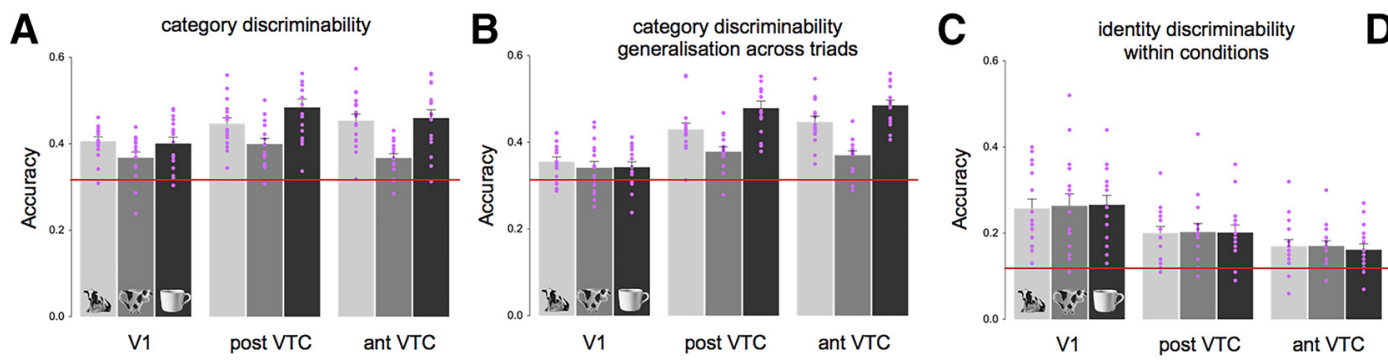

D
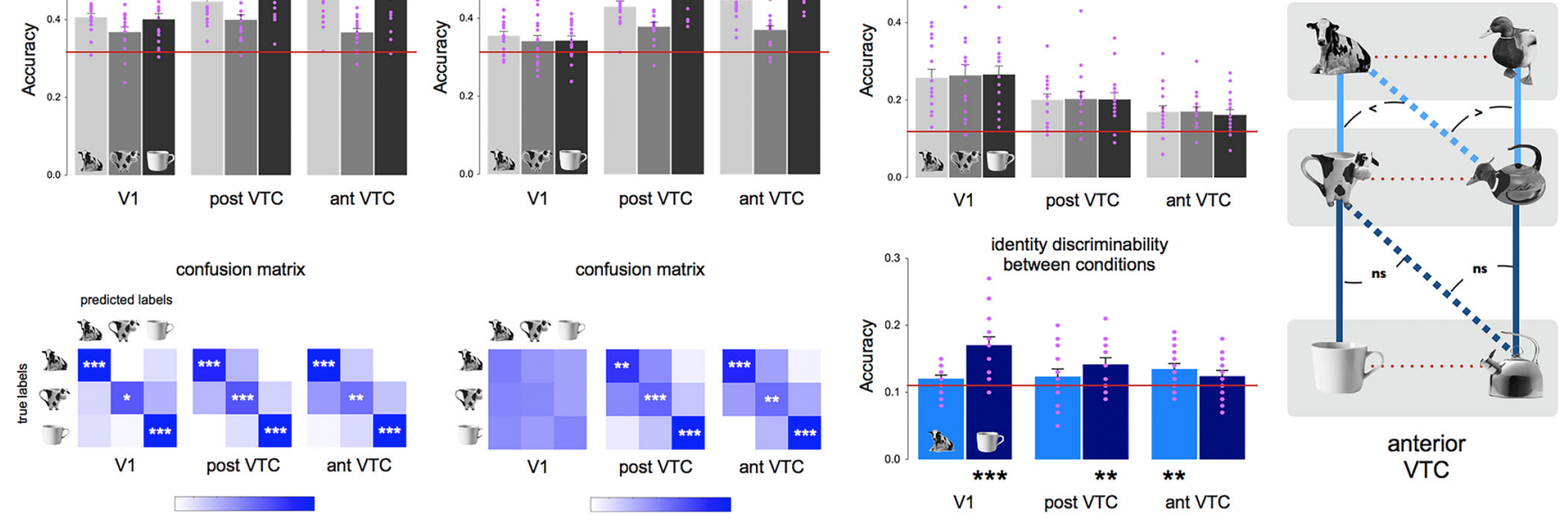

Figure 9. Classification analysis. Shown are decoding results for category discriminability ( $\boldsymbol{A}$; leave-one-run-out) and its generalization across triads ( $\boldsymbol{B}$; leave-one-triad-out) for animals, lookalikes, and objects in the three ROls. The red line shows chance level. The confusion matrix (bottom) shows classification errors between conditions. The color scale from white (low) to blue (high) indicates classification predictions. C, Stimulus identity classification within- (top) and between-conditions (bottom). D, Summary representational geometry in ant-VTC based on results from classification analyses. Gray underlays indicate clusters derived from results shown in $\boldsymbol{A}$. Shorter distance between the clusters (animals and lookalikes) indicates higher confusability derived from analysis on the confusion matrix. Within-condition stimulus identity discriminability $(\boldsymbol{C}$, top) is shown with red dotted lines. Stimulus identity generalization across conditions ( $\boldsymbol{C}$, bottom) is shown with light blue (lookalikes and animals) and dark blue (lookalikes and objects) solid lines. Significant and nonsignificant generalization of stimulus identity across conditions is show with " $<$ " and "ns," respectively. Individual participant's $(n=16)$ data points are shown in purple. Error bars indicate SEM. Asterisks $\left({ }^{* * *} p<0.001,{ }^{* *}<p<0.01,{ }^{*}<p<0.05\right)$ indicate significant values relative to chance level.

generalization across triads revealing discriminability for all three categories in post-VTC and ant-VTC $\left(t_{(15)}<4.01 ; p<0.001\right.$, for all conditions), significantly less discriminability for lookalikes relative to animals and objects (post-VTC: $t_{(15)}<3.13, p<0.007$; ant-VTC: $t_{(15)}<4.31, p<0.001$, for both conditions), and significantly higher confusability between animals and lookalikes, as opposed to objects and lookalikes, which however was significant in post-VTC only (post-VTC: $t_{(15)}=3.00, p=0.009$; ant-VTC: $p=0.14)$. As expected, due to visual differences among items in the different triads, generalization across triads did not reach significance in V1 ( $p>0.05$, for all conditions).

To replicate results reported in Figure 8, stimulus identity discriminability for neural data (Fig. 9C) was tested separately for each condition (e.g., recognizing a cow among other animals) and across conditions (e.g., matching a cow mug to cow or to a mug). Within-condition stimulus discriminability was significant for all three conditions in all ROIs $\left(t_{(15)}<3.88, p<0.001\right)$. Further, replicating correlational-based MVPA, in ant-VTC, LDA revealed generalization of stimulus identity between lookalikes and animals $\left(t_{(15)}=3.12, p=0.007\right)$, but not between lookalikes and objects ( $p>0.17$; Fig. $9 C$, bottom; note strong similarities with results reported in Fig. 8A). Instead, due to higher low-level similarities, stimulus identity generalization across lookalikes and objects was significant in more posterior areas $\left(\mathrm{V} 1: t_{(15)}=4.85, p<0.001\right.$; post-VTC: $t_{(15)}=3.21, p=$ $0.006)$.

The decoding analysis fully replicate findings reported with correlation distance despite the many differences between the two types of analyses. An overview of decoding results is shown in Figure 9D.

\section{Discussion}

With a stimulus set that specifically dissociates object appearance from object category, we investigated the characteristics of the previously reported organization of object representations in the superordinate (category) distinction of animate versus inanimate in the human brain, DNNs, and behavior. Human behavioral judgments and neural networks privilege animacy over appearance. Conversely, representations in ventral occipitotemporal cortex mostly reflect object appearance. Thus, although DNNs can largely predict human behavior, representations in ventral occipitotemporal cortex deviate from behavior and neural network representations.

Our results can be summarized as follows. First, representational content in VTC reflects animal appearance more than object identity and animacy; even though the mug and the cow mug share many high-level properties (e.g., object identity, size, function, manipulation), as well as low-level properties (e.g., shape and texture), VTC represents a cow mug closer to a real cow than to a mug. Second, VTC representations are not explained by either human perception or DNNs, which were not deceived by object appearance, and judged a cow mug being closer to a mug as opposed to a real cow. Third, given its preference in favor of animal appearance, VTC representations are remarkably poor in providing information about object identity, that is, to reflect that a cow mug is a mug. This is not a desirable property for a "what" pathway that, according to uniformly held views in visual neuroscience (DiCarlo et al., 2012), builds up representations that sustain reliable and transformation-invariant object identification and categorization. Fourth, VTC representations are not modulated by task demand and the similarity in responses to animals and lookalike objects persists even when participants performed a task requiring focusing on object animacy.

The animacy division is considered one of the main organizational principles in visual cortex (Grill-Spector and Weiner, 2014), but information content underlying this division is highly debated (Baldassi et al., 2013; Grill-Spector and Weiner, 2014; Nasr et al., 2014; Bracci and Op de Beeck, 2016; Bracci et al., 2017b; Kalfas et al., 2017). Animacy and other category distinc- 
tions are often correlated with a range of low- and higher-level visual features such as the spatial frequency spectrum (Nasr et al., 2014; Rice et al., 2014) and shape (Cohen et al., 2014; Jozwik et al., 2016), but the animacy structure remains even when dissociated from such features (Proklova et al., 2016). Here, we question what we imply with animacy. Previous reports suggest that the extent to which an object is perceived as being alive and animate is reflected in VTC representations, giving rise to a continuum where those animals perceived more animate (e.g., primates) are closely represented to humans, whereas those animals perceived less animate (e.g., bugs) are represented away from humans and closer to inanimate objects (Connolly et al., 2012; Sha et al., 2015). Contrary to this prediction, our results suggest that information content underlying the animacy organization does not relate to the animacy concept: whether an object is perceived as being alive and animate is close to irrelevant. Instead, what mostly matters is animal appearance; that is, whether an inanimate object lookalikes and shares high-level visual features with animals. Indeed, in VTC, inanimate objects such as a mug, a water kettle, or a pair of slippers with animal features (e.g., eyes, mouth, tail) are represented close to living animals (Figs. 4, 5). Even though we did not specifically include the animacy continuum in our experimental design, our design emphasizes categorical divisions. These results are not in conflict with, but rather are consistent with, the animacy continuum: bugs might be perceived less animate than dogs and cats and represented far away from humans in the animacy space because their typical facial features (eyes, mouth, etc.) are less prominent. Future studies are needed to address this possibility.

Does this mean that it is all about animal appearance? Probably not; our results showed that VTC representational content can be explained by both models, though uncorrelated $(r=0.07$; Fig. 4), and carries enough information to distinguish between real animals and lookalikes (Fig. 7). What we suggest is that information about animals in VTC is overrepresented relative to information about objects to the extent that even inanimate objects, if having animal appearance, are represented similarly to animate entities. A VTC preference toward animal representations was further supported by results showing significant information for animal's identity, which was not observed for objects; that is, VTC representations contain information to discriminate that a duck-shaped water kettle represents a duck, but not to discriminate that it is a kettle (Figs. 8, 9D). An everyday life example of this preference is our strong predisposition to perceive faces (and animals) in meaningless patterns such as toast or clouds (pareidolia), which results in face-like activations in the fusiform face area (Hadjikhani et al., 2009). To our knowledge, similar effects reported for inanimate objects are rare. We could speculate that having a system devoted to detecting animate entities and biased to represent similarly animals and inanimate objects that resemble animals might have been beneficial from an evolutionary perspective. The chance for our ancestors to survive in the wild necessitated fast and successful detection of predators. Such a system would have been advantageous: it is better to mistake a bush for a lion than the other way around. Thus, instead of inferring that VTC is fooled by the lookalike stimuli, one could also phrase the same finding more positively: VTC has the special ability to relate the lookalikes to the corresponding animal. This ability to represent lookalikes similarly to real animals might be the result of an evolution-long "training," similarly to the way, in artificial intelligence, neural networks, trained to recognize animals, start to detect animals in random-noise images (Szegedy et al., 2015).
This speculation points to one possible explanation for a particularly surprising aspect of our findings, namely the discrepancy in representations between VTC and artificial DNN. Indeed, whereas DNNs were able to predict aspects of human perception such as setting apart animals from inanimate objects (Fig. 6) and discriminating both animal and object identity (Fig. 8), contrary to recent findings (Cadieu et al., 2014; Güçlü and van Gerven, 2014; Khaligh-Razavi and Kriegeskorte, 2014; Yamins et al., 2014), our results show that DNNs representations (at the higher processing layers) do not predict human visual cortex representations. It is noteworthy that the training history of the DNNs was focused upon the categorization of a large number of categories and was not specifically biased toward, for example, animate objects. This training history might explain why DNNs are very good at identifying a cow mug as being a mug. As an example, networks trained with images of animals, when asked to overinterpret their features, similar to the perceptual phenomena of pareidolia, will start seeing animals out of random shapes such as clouds (https://ai.googleblog.com/2015/06/inceptionism-goingdeeper-into-neural.html). Given the critical role of DNN training, it is therefore noteworthy that in the absence of any specific training, there was evidence for stimulus identity generalization between animals and lookalikes (i.e., the ability to recognize a cow mug as representing a cow; Figs. $8,9 C$ ). Such a generalization is remarkable given the dominant role of image low-level features in DNN image processing (Geirhos et al., 2019).

Our results show that, across all analyses, human behavior was better predicted by DNNs than human VTC, privileging what an object is (two mugs), as opposed to its visual appearance. Information from DNNs and behavioral judgments did not show a category distinction between lookalikes and inanimate objects (e.g., all objects; Fig. 7), yet allowed object identification across changes in appearance (e.g., a cow mug is a mug; Fig. 8). These results differ from previous studies reporting some degree of correspondence between behavior and visual cortex information content (Williams et al., 2007; Carlson et al., 2014; van Bergen et al., 2015). This discrepancy might relate to the choice of task used to obtain the behavioral results that were compared with neural data; here, we used a cognitive task (similarity judgments), whereas a categorization task based on reaction times (e.g., visual search task) might have been a better predictor for visual cortex representations (Proklova et al., 2016; Cohen et al., 2017).

Finally, visual cortex representations were not modulated by tasks. Despite the different focus on object appearance in one case (appearance task) and on object identity in the other case (animacy task), the neural data were striking similar across task sessions, revealing no difference in any of the performed analyses. These results confirm and add to recent findings showing that task demand appears not to have much influence on the representational structure of ventral visual cortex (Harel et al., 2014; Bracci et al., 2017a; Bugatus et al., 2017; Hebart et al., 2018).

The mere observation that a stimulus that looks like an animal, or part of it such as a face, can elicit animal- or face-like responses in ventral visual cortex is not novel, with studies on face pareidolia being the most relevant example (Hadjikhani et al., 2009). What makes our study novel and our results striking is the availability of behavioral and neural network data that makes a very different prediction: the systematic comparison and dissociation of neural, behavioral, and DNN representations; the quantification of the degree of bias toward representing appearance rather than animacy; the persistence of this bias in VTC even when performing a task requiring to report animacy; and the 
detrimental effect of this preference upon the informational content about object identity.

To summarize, these results highlight substantial differences in the way that categorical representations in the human brain, human judgments, and DNNs characterize objects. Whereas DNN representations resemble human behavior and heavily weight object identity, representations in ventral visual cortex are very much constrained by object appearance. Based on these findings, we might have to reconsider the traditional view of the human visual cortex as a general object recognition machine like the artificial neural networks are trained to be.

\section{References}

Baldassi C, Alemi-Neissi A, Pagan M, Dicarlo JJ, Zecchina R, Zoccolan D (2013) Shape similarity, better than semantic membership, accounts for the structure of visual object representations in a population of monkey inferotemporal neurons. PLoS Comput Biol 9:e1003167.

Bracci S, Op de Beeck H (2016) Dissociations and associations between shape and category representations in the two visual pathways. J Neurosci 36:432-444.

Bracci S, Daniels N, Op de Beeck H (2017a) Task context overrules objectand category-related representational content in the human parietal cortex. Cereb Cortex 27:310-321.

Bracci S, Ritchie JB, de Beeck HO (2017b) On the partnership between neural representations of object categories and visual features in the ventral visual pathway. Neuropsychologia 105:153-164.

Brainard DH (1997) The psychophysics toolbox. Spat Vis 10:433-436.

Bugatus L, Weiner KS, Grill-Spector K (2017) Task alters category representations in prefrontal but not high-level visual cortex. Neuroimage 155: 437-449.

Cadieu CF, Hong H, Yamins DL, Pinto N, Ardila D, Solomon EA, Majaj NJ, DiCarlo JJ (2014) Deep neural networks rival the representation of primate IT cortex for core visual object recognition. PLoS Comput Biol 10:e1003963.

Caramazza A, Shelton JR (1998) Domain-specific knowledge systems in the brain the animate-inanimate distinction. J Cogn Neurosci 10:1-34.

Carlson TA, Ritchie JB, Kriegeskorte N, Durvasula S, Ma J (2014) Reaction time for object categorization is predicted by representational distance. J Cogn Neurosci 26:132-142.

Cohen MA, Konkle T, Rhee JY, Nakayama K, Alvarez GA (2014) Processing multiple visual objects is limited by overlap in neural channels. Proc Natl Acad Sci U S A 111:8955-8960.

Cohen MA, Alvarez GA, Nakayama K, Konkle T (2017) Visual search for object categories is predicted by the representational architecture of highlevel visual cortex. J Neurophysiol 117:388-402.

Connolly AC, Guntupalli JS, Gors J, Hanke M, Halchenko YO, Wu YC, Abdi H, Haxby JV (2012) The representation of biological classes in the human brain. J Neurosci 32:2608-2618.

Desimone R, Albright TD, Gross CG, Bruce C (1984) Stimulus-selective properties of inferior temporal neurons in the macaque. J Neurosci 4:2051-2062.

DiCarlo JJ, Zoccolan D, Rust NC (2012) How does the brain solve visual object recognition? Neuron 73:415-434.

Downing PE, Jiang Y, Shuman M, Kanwisher N (2001) A cortical area selective for visual processing of the human body. Science 293:2470-2473.

Gao T, Newman GE, Scholl BJ (2009) The psychophysics of chasing: a case study in the perception of animacy. Cogn Psychol 59:154-179.

Gao T, McCarthy G, Scholl BJ (2010) The wolfpack effect. perception of animacy irresistibly influences interactive behavior. Psychol Sci 21:18451853.

Geirhos R, Rubisch P, Michaelis C, Bethge M, Wichmann FA, Brendel W (2019) ImageNet-trained CNNs are biased towards texture; increasing shape bias improves accuracy and robustness. Available at https://arxiv. org/abs/1811.12231.

Grill-Spector K, Weiner KS (2014) The functional architecture of the ventral temporal cortex and its role in categorization. Nat Rev Neurosci 15: $536-548$.

Grill-Spector K, Kushnir T, Edelman S, Itzchak Y, Malach R (1998) Cueinvariant activation in object-related areas of the human occipital lobe. Neuron 21:191-202.

Grill-Spector K, Kushnir T, Edelman S, Avidan G, Itzchak Y, Malach R
(1999) Differential processing of objects under various viewing conditions in the human lateral occipital complex. Neuron 24:187-203.

Gross CG, Rocha-Miranda CE, Bender DB (1972) Visual properties of neurons in inferotemporal cortex of the macaque. J Neurophysiol 35:96-111.

Güçlü U, van Gerven MA (2014) Unsupervised feature learning improves prediction of human brain activity in response to natural images. PLoS Comput Biol 10:e1003724.

Güçlü U, van Gerven MA (2015) Deep neural networks reveal a gradient in the complexity of neural representations across the ventral stream. J Neurosci 35:10005-10014.

Hadjikhani N, Kveraga K, Naik P, Ahlfors SP (2009) Early (M170) activation of face-specific cortex by face-like objects. Neuroreport 20:403-407.

Harel A, Kravitz DJ, Baker CI (2014) Task context impacts visual object processing differentially across the cortex. Proc Natl Acad Sci U S A 111:E962-E971.

He K, Zhang X, Ren S, Sun J (2015) Delving deep into rectifiers: surpassing human-level performance on ImageNet classification. Available at https://arxiv.org/abs/1502.01852.

Hebart MN, Bankson BB, Harel A, Baker CI, Cichy RM (2018) The representational dynamics of task and object processing in humans. Elife 7:e32816.

Jozwik KM, Kriegeskorte N, Mur M (2016) Visual features as stepping stones toward semantics: explaining object similarity in IT and perception with non-negative least squares. Neuropsychologia 83:201-226.

Kalfas I, Kumar S, Vogels R (2017) Shape selectivity of middle superior temporal sulcus body patch neurons. eNeuro 4:ENEURO.0113-17.2017.

Kanwisher N, McDermott J, Chun MM (1997) The fusiform face area: a module in human extrastriate cortex specialized for face perception. J Neurosci 17:4302-4311.

Khaligh-Razavi SM, Kriegeskorte N (2014) Deep supervised, but not unsupervised, models may explain IT cortical representation. PLoS Comput Biol 10:e1003915.

Kheradpisheh SR, Ghodrati M, Ganjtabesh M, Masquelier T (2016a) Deep networks can resemble human feed-forward vision in invariant object recognition. Sci Rep 6:32672.

Kheradpisheh SR, Ghodrati M, Ganjtabesh M, Masquelier T (2016b) Humans and deep networks largely agree on which kinds of variation make object recognition harder. Front Comput Neurosci 10:92.

Konkle T, Oliva A (2012) A real-world size organization of object responses in occipitotemporal cortex. Neuron 74:1114-1124.

Kriegeskorte N (2015) Deep neural networks: a new framework for modeling biological vision and brain information processing. Annu Rev Vis Sci 1:417-446.

Kriegeskorte N, Goebel R, Bandettini P (2006) Information-based functional brain mapping. Proc Natl Acad Sci U S A 103:3863-3868.

Kriegeskorte N, Mur M, Bandettini P (2008a) Representational similarity analysis - connecting the branches of systems neuroscience. Front Syst Neurosci 2:4.

Kriegeskorte N, Mur M, Ruff DA, Kiani R, Bodurka J, Esteky H, Tanaka K, Bandettini PA (2008b) Matching categorical object representations in inferior temporal cortex of man and monkey. Neuron 60:1126-1141.

Kriegeskorte N, Mur M (2012) Inverse MDS: inferring dissimilarity structure from multiple item arrangements. Front Psychol 3:245.

Kubilius J, Bracci S, Op de Beeck HP (2016) Deep neural networks as a computational model for human shape sensitivity. PLoS Comput Biol 12:e1004896

Nasr S, Echavarria CE, Tootell RB (2014) Thinking outside the box: rectilinear shapes selectively activate scene-selective cortex. J Neurosci 34: 6721-6735.

New J, Cosmides L, Tooby J (2007) Category-specific attention for animals reflects ancestral priorities, not expertise. Proc Natl Acad Sci U S A 104: $16598-16603$.

Oosterhof NN, Connolly AC, Haxby JV (2016) CoSMoMVPA: multimodal multivariate pattern analysis of neuroimaging data in Matlab/ GNU octave. Front Neuroinform 10:27.

Op de Beeck HP (2010) Against hyperacuity in brain reading: spatial smoothing does not hurt multivariate fMRI analyses? Neuroimage 49: 1943-1948.

Op de Beeck HP, Torfs K, Wagemans J (2008) Perceived shape similarity among unfamiliar objects and the organization of the human object vision pathway. J Neurosci 28:10111-10123.

Op de Beeck HP, Brants M, Baeck A, Wagemans J (2010) Distributed sub- 
ordinate specificity for bodies, faces, and buildings in human ventral visual cortex. Neuroimage 49:3414-3425.

Proklova D, Kaiser D, Peelen MV (2016) Disentangling representations of object shape and object category in human visual cortex: the animateinanimate distinction. J Cogn Neurosci 28:680-692.

Rice GE, Watson DM, Hartley T, Andrews TJ (2014) Low-level image properties of visual objects predict patterns of neural response across categoryselective regions of the ventral visual pathway. J Neurosci 34:8837-8844.

Ritchie JB, Bracci S, Op de Beeck H (2017) Avoiding illusory effects in representational similarity analysis: what (not) to do with the diagonal. Neuroimage 148:197-200.

Russakovsky O, Deng J, Su H, Krause J, Satheesh S, Ma S, Huang Z, Karpathy A, Khosla A, Bernstein M, Berg A, Fei-Fei L (2014) ImageNet large scale visual recognition challenge. Available at https://arxiv.org/abs/1409. $0575 \mathrm{v} 3$.

Scholl BJ, Gao T (2013) Perceiving animacy and intentionality: Visual processing or higher-level judgment? In: Social perception: Detection and interpretation of animacy, agency, and intention (Rutherford MD, Kuhlmeier VA eds), pp. 197-230. Cambridge, MA: MIT Press.

Sha L, Haxby JV, Abdi H, Guntupalli JS, Oosterhof NN, Halchenko YO, Connolly AC (2015) The animacy continuum in the human ventral vision pathway. J Cogn Neurosci 27:665-678.

Simonyan K, Zisserman A (2014) Very deep convolutional networks for large-scale image recognition. Available at https://arxiv.org/abs/1409. 1556.
Smith SM, Nichols TE (2009) Threshold-free cluster enhancement: addressing problems of smoothing, threshold dependence and localisation in cluster inference. Neuroimage 44:83-98.

Szegedy C, Liu W, Jia Y, Sermanet P, Reed S, Anguelov D, Erhan D, Vanhoucke V, Rabinovich A (2015) Going deeper with convolutions. Available at https://arxiv.org/abs/1409.4842v1.

Tremoulet PD, Feldman J (2000) Perception of animacy from the motion of a single object. Perception 29:943-951.

van Bergen RS, Ma WJ, Pratte MS, Jehee JF (2015) Sensory uncertainty decoded from visual cortex predicts behavior. Nat Neurosci 18: $1728-1730$.

Vedaldi A, Lenc K (2016) MatConvNet: Convolutional Neural Networks for MATLAB. Available at https://arxiv.org/abs/1412.4564.

Walther A, Nili H, Ejaz N, Alink A, Kriegeskorte N, Diedrichsen J (2016) Reliability of dissimilarity measures for multi-voxel pattern analysis. Neuroimage 137:188-200.

Williams MA, Dang S, Kanwisher NG (2007) Only some spatial patterns of fMRI response are read out in task performance. Nat Neurosci 10:685686.

Xia M, Wang J, He Y (2013) BrainNet viewer: a network visualization tool for human brain connectomics. PLoS One 8:e68910.

Yamins DL, Hong H, Cadieu CF, Solomon EA, Seibert D, DiCarlo JJ (2014) Performance-optimized hierarchical models predict neural responses in higher visual cortex. Proc Natl Acad Sci U S A 111:8619-8624. 